Original Article (short paper)

\title{
Postural Control of People in treatment for substance use disorder
}

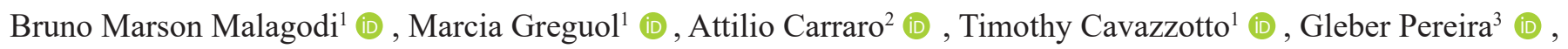 \\ Helio Serassuelo Junior ${ }^{1}$ \\ ${ }^{1}$ Universidade Estadual de Londrina, Educação Física, Londrina, PR, Brasil, ${ }^{2}$ Libera Universita' di Bol- \\ zano, Bolzano, Trentino-Alto Adige, Italy; ${ }^{3}$ Universidade Federal do Paraná, Curitiba, PR, Brasil
}

\begin{abstract}
Aims: One of the common sequelae of chronic abuse of alcohol and/or illicit drugs is the impairment of body balance control, caused by long-term neurological damage. This study aimed to investigate the postural control of individuals hospitalized for the treatment of substance use disorder (initial phase) and to compare the results obtained by a control group. Method: For this, One-hundred fourteen individuals hospitalized for the treatment of substance use disorder and eighty-eight healthy controls, all males, were analyzed. Body mass, height, and waist circumference were measured. Were performed a balance test in a static upright position with feet side by side using a plantar pressure platform. Results: Individuals with substance use disorder have shown significantly lower results on body balance compared with controls. Individuals with chemical dependence showed balance results open-eyes, similar/lower than control subjects with close-eyes. Still, those who used only alcohol or alcohol combined with other illicit drugs presented worse results. Finally, impairments in body balance variables showed a significant correlation with age and substance time of use. Conclusion: Even in the early stage of treatment, substance use disorder considerably impairs the balance of the investigated men. The worst results were found in alcohol-dependents (alcohol alone or alcohol combined with other drugs).
\end{abstract}

Keywords: Alcohol, Illicit drugs, Chemical Dependence, Body Balance, Postural Control.

\section{Introduction}

Chronic and excessive consumption of alcohol and illicit drugs can impair several central nervous system functions, including cognitive and motor aspects of individuals who misuse these substances. The acute effect of alcohol and other drugs on postural control is well established in the literature ${ }^{1,2}$. Deficits in postural control and balance, as well as adverse sedative effects, such as impaired cognitive functioning and muscle relaxation, are reversible after cessation (withdrawal) of the abuse of these psychotropic drugs ${ }^{1,3,4}$.

According to Carlini et al. ${ }^{5}$, data from the last household survey on the use of psychotropic drugs in Brazil showed that $22.8 \%$ of the population surveyed has already used illicit drugs. Lifetime marijuana use appears first among illicit drugs, with $8.8 \%$ of respondents, and cocaine use was reported by $2.9 \%$ of the individuals interviewed. Alcohol use was reported by $74.6 \%$ of the interviewees, and the prevalence of individuals classified as a dependent was $12.3 \%$.

The treatment procedures for substance use disorders (SUD) involve psychological and/or pharmacological support and an extended follow-up period ${ }^{4}$. Psychological approaches used in the treatment of alcohol and drug dependence may include mutual help groups, group therapy, and psychotherapy, as well as short- or long-term hospitalization in specialized institutions for the treatment of chemical dependence ${ }^{6}$. Chemical dependence can lead to several impairments to the individual's mobility, including impairments in their postural control ${ }^{7,8}$.

Postural control can be defined as the act of maintaining, reaching, or restoring a state of balance during any posture or activity. Adequate postural control is essential for daily activities and requires the integration of visual, vestibular, and proprioceptive information ${ }^{9,10}$. Individuals with chronic SUD with varying periods of abstinence presented lower levels of balance and coordination than control groups in different sensory conditions analyzed, both in static and dynamic situations ${ }^{2,10,11}$.

Few studies have been conducted to investigate postural control in individuals initiating the recovery process and the harm caused by the long-term interaction of alcohol and other illicit drug abuse ${ }^{12}$. The main cause of this scarcity is the poor adherence 
to treatment and subsequent maintenance of the recovery process, making it difficult to follow the patient for long periods and gain a better understanding of the mechanisms involved in the neural plasticity that occurs during the sobriety process after years of excessive alcohol and other drug consumption ${ }^{7}$.

Most studies on body balance in individuals with SUD have been conducted with individuals with alcoholism, leaving a gap on the effects of other drugs on this variable. Alcohol dependence has been associated with a decrease in postural control in more than $60 \%$ of abstinent patients, suggesting that this deficiency is directly related to alcohol consumption throughout life ${ }^{2}$. Thus, it can be assumed that the negative impacts on postural control in these patients may have been caused, mainly, by the toxic effect related to the dose of alcohol consumed, that is, the greater the amount and time of exposure to addiction, the greater the losses in postural control.

The study conducted by Schmidt et al. ${ }^{13}$ using dynamic posturography also concluded that alcohol consumption has a deleterious effect on the body balance of abstinent alcoholic individuals and that these individuals present significant alterations in postural balance when compared to non-alcoholics. Moreira et al. ${ }^{14}$ in their study using posturography, highlight that time of use of illicit drugs demonstrated a significant and positive correlation with oscillation velocity in the closed eyes condition. Thus, the longer the use of illicit drugs, the greater the individual's difficulty in maintaining body balance when visual and proprioceptive information was absent or distorted.

Some variables that intervene in SUD, such as subjects' age, time of consumption, and type of substance consumed (alcohol or other illicit drugs) may interfere with postural control. In addition, deficits in postural control mechanisms in individuals who are dependent on different drugs are not well known. Thus, this study aims to investigate postural control of men in treatment for SUD (initial phase) and to compare these results with those of healthy control subjects. In addition, individuals who use alcohol and other illicit drugs will also be compared.

\section{Methods}

\section{Sample}

The study was approved by the local Ethics Committee (number 2.125.747/2017). We evaluated one-hundred fourteeen adult males diagnosed with Substance Use Disorders (SUD), aged from $18-59$ years $(34.9 \pm 12.2$ years $)$, hospitalized for treatment in a private clinic in south Brazil. The clinic had a multidisciplinary approach to the treatment of chemical dependency, offering non-pharmacological and pharmacological therapy when necessary. Only individuals who were in the initial phase of treatment (after the first two weeks of hospitalization) and who presented at least one year of dependence diagnosis were included in the sample. Since withdrawal symptoms may occur in the first two weeks, the subjects were evaluated on the 15 th day of hospitalization. Exclusion criteria were having a motor or cognitive impairment that hamper participation in motor tests and understanding of assessment tools (e.g. excessive tremble or Mini-Mental State Examination $<21$ ). Individuals with acute withdrawal symptoms were excluded from the study. The institution physician consulted all the patients before the test sessions and only those considered fit to participate in the research were included.

In the control group (CG), eighty-eight healthy adult male participants with a mean age of $35.3 \pm 12$ years and without a chemical dependence diagnosis were evaluated, selected from the local university community. These individuals were in the same age group and presented similar anthropometric characteristics to the clinical sample. Individuals were excluded if they reported the use of illicit psychotropic substances (illicit drugs) and alcohol abuse - 4 (four) or more times a week, or consumption of 5 (five) or more doses on a single occasion during the 30 days preceding the study ${ }^{15}$. A sample size of 82 individuals for each group was estimated by the $\mathrm{G}$ * Power 3.1.9 software for power $(1-\beta)$ of $80 \%$, a one-tailed significance level $(\alpha)$ of .05 , and high effect size $(\mathrm{d}=.8)$. All the participants signed an Informed Consent, agreeing to participate in the research

\section{Procedure}

Initially, all the individuals answered an anamnesis with information about age, schooling, type of substance consumed, time of use/abuse, medication use, and regular participation in a physical exercise program in the month preceding hospitalization. Measurements of anthropometric variables, weight, height, and waist circumference were collected, and BMI was calculated.

Analysis of postural control was conducted through a balance test in a static upright position with feet side by side using the plantar pressure platform myoPressure Noraxon $\AA$. During the test individuals remained in the bipodal position for 30 seconds in two conditions - eyes open and eyes closed - and data were recorded, stored, and then analyzed using Noraxon ${ }^{\circledR}$ myoRESEARCH 3.10 software. To evaluate the body balance of the participants, COP (center of pressure) path length (the distance traveled by the COP in millimeters during the analyzed time) and the ellipse area (the area covered by the center of pressure in the mediolateral and anteroposterior axes) were verified. The lower the values recorded, the better the balance.

\section{Statistics}

Descriptive statistics were calculated, one-way ANOVA was used to compare body balance variables between groups, and a generalized linear model (GLM) was used to adjust differences according to age and physical exercise practice in the month preceding hospitalization (YES or NO). Effect size between groups (Cohen's d) was calculated using the equation: Cohen's $\mathrm{d}=$ Mean1 - Mean2/Standard Deviation ${ }_{\text {pooled }}$. The effect size was interpreted as follows: $\mathrm{d}<0.20$ very small, $\mathrm{d}=0.20-0.59$ small, $\mathrm{d}=0.60-1.19$ medium, $\mathrm{d}=1.20-1.99$ large, $\mathrm{d} \geq 2.00$ very large, according to the recommendations of Hopkins et al. ${ }^{16}$. 
Pearson's correlation test was applied to verify the relationships between body balance variables, age, and time of substance use. Significance was set at $p \leq 0.05$.

\section{Results}

Among participants with SUD, 31 subjects (27.2\%) reported regular use of alcohol, 21 (18.4\%) use of cocaine/crack, and 62 (54.4\%) reported polysubstance use (alcohol + illicit drugs). The mean time of consumption of the substance was 13.97 \pm 1.13 years. In this variable, the group of alcohol dependents presented significantly longer consumption time $(22.87 \pm 14.4$ years) than cocaine/crack $(10.14 \pm 7.3)$ or polysubstance $(10.82$ \pm 7.4 ) users. Despite the great variability in the data, 68 subjects from the SUD group (59.9\%) stated that they had been using the substance for at least 10 years. Regarding the level of schooling, 62 subjects (54.4\%) with SUD reported elementary education, while only $14(12.3 \%)$ reported higher education.

When questioned about physical exercise, 42 subjects (47.7\%) from the CG and 25 (21.9\%) from the SUD group stated that they had participated in a regular and supervised program in the previous month. There were no significant differences between age and BMI values of the participants from both groups $(p>.05)$. Among individuals with SUD, alcohol users had a higher average age than drug or alcohol + drug (polysubstance) users. Participants' descriptive data are summarized in table 1.

It was verified that the group of individuals with alcohol-dependence was significantly older when compared with cocaine/crack and polysubstance users $(\mathrm{F}=22.51 ; p<.001)$. No differences were observed in BMI. Comparisons between groups (SUD and CG) related to the variables of body balance are summarized in Figure 1.

Table 1. - Descriptive data of study participants.

\begin{tabular}{|c|c|c|c|c|}
\hline & \multirow{2}{*}{$\begin{array}{c}C G \\
(N=88)\end{array}$} & \multicolumn{3}{|c|}{ SUD GROUP } \\
\hline & & $\begin{array}{l}\underline{\text { Alcohol }} \\
(\mathbf{N}=31)\end{array}$ & $\begin{array}{c}\text { Cocaine/Crack } \\
(\mathbf{N}=21)\end{array}$ & $\begin{array}{l}\text { Polysubstance } \\
\quad(N=62)\end{array}$ \\
\hline & $\mathbf{M} \pm \mathbf{S D}$ & $\mathbf{M} \pm \mathbf{S D}$ & $\mathbf{M} \pm \mathbf{S D}$ & $\mathbf{M} \pm \mathbf{S D}$ \\
\hline Age (years) & $34.9 \pm 12.2$ & $45.3 \pm 9.3$ & $35.5 \pm 11$ & $30.2 \pm 8.7$ \\
\hline BMI $\left(\mathrm{kg} / \mathrm{m}^{2}\right)$ & $25.91 \pm 3.55$ & $24.92 \pm 3.7$ & $25.06 \pm 2.98$ & $24.88 \pm 3.9$ \\
\hline COP PATH OE (mm) & $139.96 \pm 11.11$ & $281.87 \pm 17.16$ & $174.43 \pm 20.85$ & $202.06 \pm 12.14$ \\
\hline COP PATH CE (mm) & $218.87 \pm 41.53$ & $599.9 \pm 64.53$ & $288.81 \pm 78.41$ & $317.82 \pm 45.63$ \\
\hline ELLIPSE OE $\left(\mathrm{mm}^{2}\right)$ & $98.12 \pm 11.39$ & $235.26 \pm 18.15$ & $153.9 \pm 22.05$ & $164.81 \pm 12.84$ \\
\hline ELLIPSE CE $\left(\mathrm{mm}^{2}\right)$ & $127.72 \pm 37.38$ & $463.13 \pm 58.89$ & $237.95 \pm 71.55$ & $248.5 \pm 41.64$ \\
\hline
\end{tabular}

Note: $\mathrm{M}=$ Mean; $\mathrm{SD}=$ Standard deviation; $\mathrm{OE}=$ Open eyes; $\mathrm{CE}=$ closed eyes; $\mathrm{BMI}=$ body mass index; $\mathrm{COP}=$ center of pressure
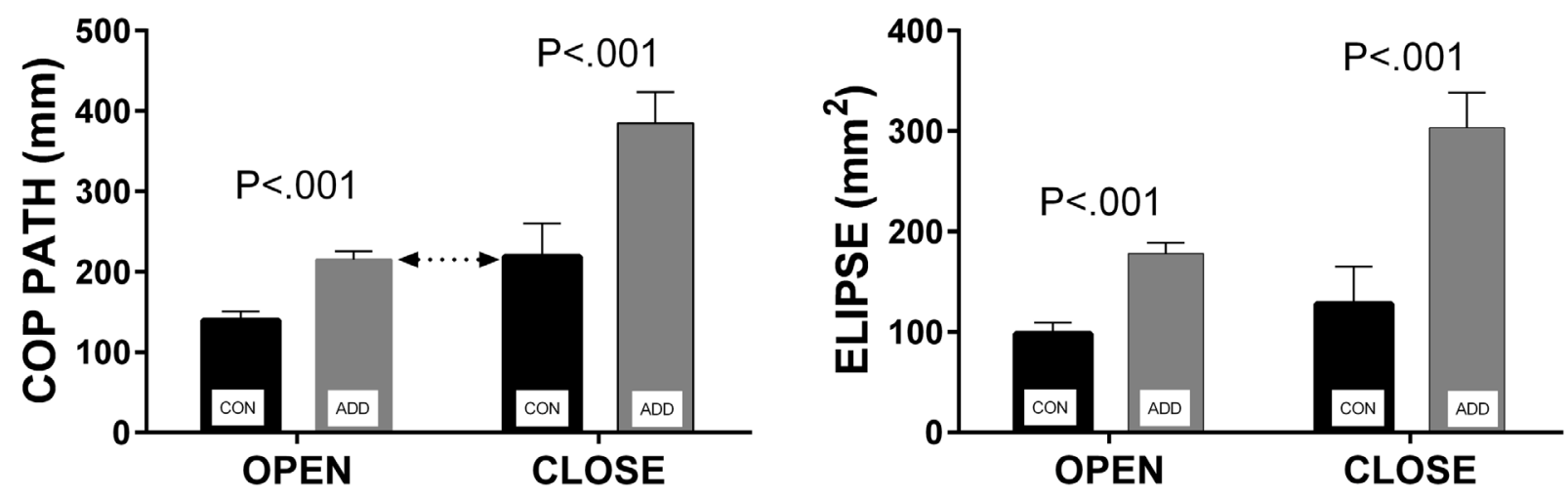

Figure 1. Body balance analysis in substance disorders group (SUD) and control group (CG).

(Note: GENLIN differences adjusted by Age, Physical Exercise) 
The data obtained show that subjects with SUD presented significantly worse values for body balance than the $\mathrm{CG}$, in both open and closed eye conditions (COP Path OE: F = 31,57, $\mathrm{p}<0.001$; COP Path CE: $\mathrm{F}=10,58, \mathrm{p}<0.001$; Ellipse OE: $\mathrm{F}$ $=29,98, p<0.001$; Ellipse CE: $F=13,16, p<.001)$. Figure 2 presents the observed effect sizes for both groups and in both conditions (open and closed eyes). It is possible to highlight very large effects in the comparisons between groups, showing that the $C G$ presented lower values for the body balance variables $(\mathrm{ES}=-4.12$ for COP Path Open Eyes and $\mathrm{ES}=-7.0$ for COP Path Closed Eyes; ES= -4.8 for Ellipse Open Eyes and $\mathrm{ES}=-7.2$ for Ellipse Closed Eyes). In the comparisons between conditions, the effects were again very large for the SUD group, with lower values in the open eyes condition. For the CG, a very large effect was observed in the COP Path variable and a medium effect in the Ellipse variable.

When the SUD group was analyzed according to the type of substance consumed, different behaviors were observed in the balance variables, as shown in Figures $3 \mathrm{a}$ and $3 \mathrm{~b}$. In both open and closed eyes conditions, only the alcohol and polysubstance groups had higher values than the CG for COP Path and Ellipse. In addition, the alcohol group presented higher values than the cocaine/crack and polysubstance groups, except for the Ellipse in the closed eyes condition.

Finally, significant correlations were observed in the open eyes situation between COP Path and age $(r=.19 ; p=.045)$, ellipse area and age $(r=.19 ; p=.040)$, COP Path and time of substance consumption $(r=.34 ; p=.000)$, and ellipse area and time of substance consumption $(r=.25 ; p=.008)$.
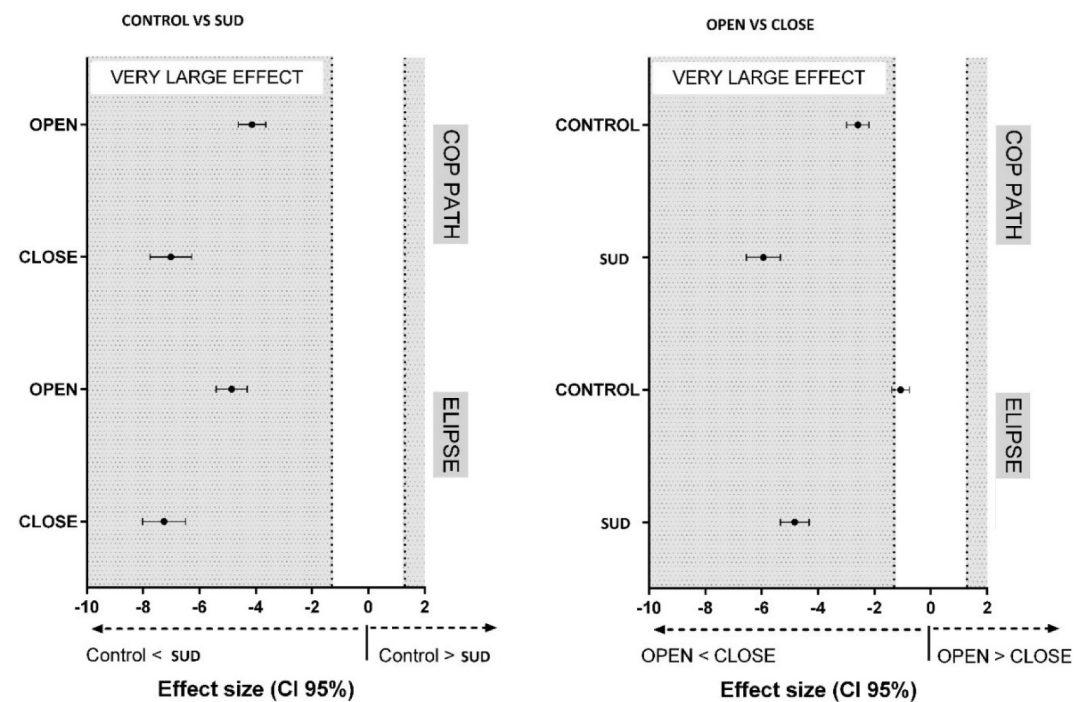

Figure 2. Effect size observed in comparisons between groups (CG X SUD) and between conditions (Open X Closed eyes).
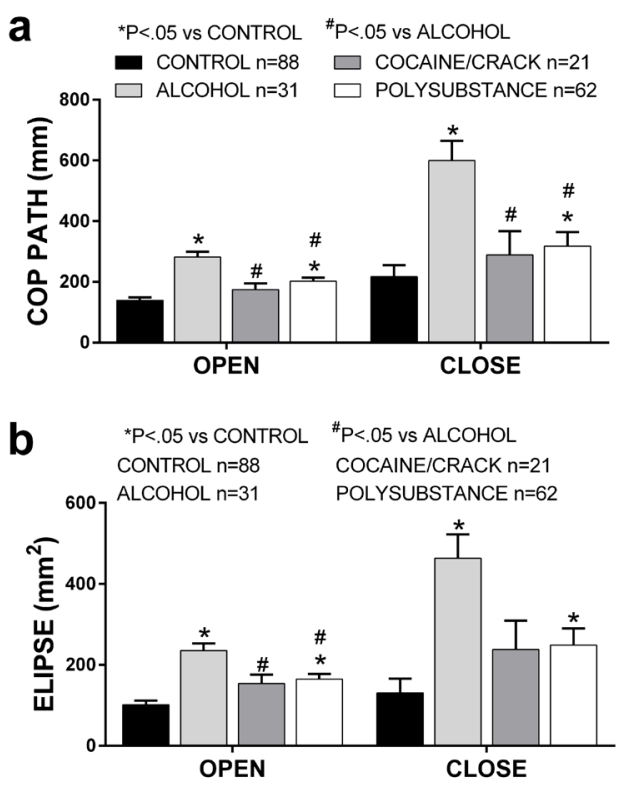

Figure 3. COP Path (3a) and Ellipse area (3b) values in the different groups in open and closed eyes conditions. 


\section{Discussion}

The study aimed to compare data related to body balance between individuals in treatment for SUD and control individuals with no history of substance abuse. In the descriptive analysis of the participants with SUD, it was possible to verify a high prevalence of individuals dependent on polysubstance, with at least 10 years of consumption, and a low level of education. According to the World Health Organization ${ }^{17}$, the onset of dependence is early, which usually leads to dropping out of school at younger ages, helping to explain the low level of schooling found ${ }^{12}$.

Another point observed was the low prevalence of individuals with SUD who had practiced regular physical exercise in the month preceding hospitalization when compared to the $\mathrm{CG}$. The data should be interpreted with caution since some sequelae of chronic SUD, such as weakness, and motor coordination and balance problems can reduce participation in physical exercise programs $^{18-20}$. In addition, the practice or not of physical exercise in the present study was only reported by the individual. Despite this, other studies in the literature have demonstrated a higher prevalence of sedentary behaviors among individuals with SUD, especially among those with a longer time of use $\mathrm{e}^{21,22}$.

When both groups were compared, the CG presented higher results in the COP Path and Ellipse area, denoting worse body balance control. These worse results of the SUD group were seen in both open and closed eye conditions. In addition, as highlighted in figure 1, the SUD group with eyes open presented results similar to those of the CG with eyes closed for the variable COP Path.

It is known that the consumption of alcohol and/or illicit drugs can negatively affect body balance ${ }^{8,12,14}$, which may be due to the increase in the occurrence of tremors and excessive body oscillation ${ }^{1,23,24}$, damage to structures of the central and peripheral nervous system ${ }^{13}$, or, in the specific case of alcohol consumption, cerebellar damage characterized by degeneration and loss of volume of Purkinje cells ${ }^{25}$. Although the practice of physical exercise was considered as a covariant for the comparison between groups, it is known that individuals with SUD, in addition to neurological damage, may present low levels of muscular strength when compared to people without dependence, a factor which may also negatively influence the results ${ }^{7,19}$.

When comparing the users by the type of illicit drug consumed, it was verified that those with alcohol dependence were significantly older and had a long time of dependence. This fact may mean that alcohol dependents take longer to seek treatment since alcohol is a drug considered "acceptable" and licit by society ${ }^{24}$.

Likewise, the subgroup of alcohol-dependent individuals, when compared to cocaine/crack or polysubstance (alcohol + illicit drugs) dependents, presented greater impairments in body balance. Furthermore, the polysubstance dependent group demonstrated significantly worse values in the body balance variables when compared to the $\mathrm{CG}$, which was not observed for the cocaine/crack group. These differences reinforce the potential impairment that abusive alcohol consumption can bring to the control of body balance. It should be noted, however, that the alcohol-dependent group presented a significantly higher mean age and substance consumption time than the other groups.

The results found in the present study diverge somewhat from those seen by Fein, Smith, and Greenstein ${ }^{12}$, who compared alcohol-dependent, alcohol + illicit drug dependent, and control individuals and showed that the alcohol + illicit drug group presented the worst results in body balance. However, in this study, individuals in the alcohol + drug group also presented longer substance use time, which may have interfered with the results.

Another study by Moreira, Ganança, and Caovila ${ }^{14}$ comparing alcohol-dependent, illicit drug-dependent, and control individuals found that the first two groups had worse results in body balance. However, as the specific types of drug consumed, as well as the consumption time, was not specified in this study, comparisons with our results are hampered.

It is known that the consumption time of the substance is a factor that negatively affects balance control $^{26}$, especially in the case of alcohol. Wöber et al. ${ }^{2}$ analyzed 82 alcohol-dependent individuals who had abstained less than one month previously and found that they demonstrated worse results in balance tests when compared to controls. The authors also found a positive relationship between the damage to balance and the time of alcohol consumption, reinforcing that chronic dependence can cause severe impairment over the years.

In the specific case of the present study, a positive relationship was also observed between impaired balance, age, and duration of substance use, indicating that the older the individual and the longer the consumption time, the higher the values measured in the COP path and ellipse area. It would be possible to speculate that alcohol-dependent individuals presented the worst results only because they were older, however, the polysubstance group (alcohol + illicit drugs) also presented lower values in the body balance variables when compared to the control, despite having a lower mean age. In this way, it seems that the use of alcohol, combined or not with other illicit drugs, is an independent factor for damage to body balance and that this impairment is greater the longer the consumption time of the substance.

Regarding the withdrawal time, in our study this variable was not analyzed since all the participants had been in rehabilitation for two weeks, that is, they had a very short period of abstinence. Nevertheless, it is known that prolonged periods of abstinence can reverse some of the damage caused by abusive consumption of substances to body balance. Fein and Greenstein ${ }^{10}$ followed individuals with chronic alcoholism who were abstainers for 6 weeks and one year, comparing their body balance to a CG. However, even after one year of abstinence, the authors did not find improvements in body balance, noting that it would probably take longer without substance use to observe any improvement. Rosenbloon et al. ${ }^{3}$, following the body balance of individuals with alcoholism over two years of abstinence, observed that after this period there were significant improvements in the analyzed variables. These findings indicate that long periods without the use of the substance are necessary so that beneficial effects can be verified in body balance.

Our study presents some limitations, such as the unequal distribution in the subgroups by type of substance consumed, as well as the type of instruments applied to analyze body balance, 
which evaluates only the static upright position and to assess the physical exercise practice. Nevertheless, it is possible to highlight that the verified data can provide relevant subsidies for professionals working in health services that provide care for individuals with SUD, to emphasize the importance of intervention programs aimed at improving the balance of these individuals, supporting their recovery process and reducing the risk of motor impairments.

\section{Conclusions}

From the data analyzed, it is observed that the balance of individuals with SUD was significantly impaired when compared to control individuals. In addition, those who used only alcohol or alcohol combined with other illicit drugs presented worse results compared to cocaine/crack users. Finally, impairments in body balance variables presented a significant correlation with age and duration of substance use. In this way, the importance of interventions as early as possible is reinforced, in order to avoid prolonged use of substances, in addition to intervention programs aimed at improving or maintaining body balance.

The increasing number of people in SUD situations in Brazil imposes an imminent need for more in-depth information about their health status so that professionals from different areas can carry out interventions in a specific and grounded way. Thus, the importance of new studies with this population is evidenced, with interventions involving protocols of physical exercises, analyses of other variables such as muscle strength, as well as larger and more homogeneous samples, in order to obtain reliable data about the balance of these individuals.

\section{References}

1. De Groot MH, Van Campen JP, Moek MA, Tulner LR, Beijnen JH, Lamoth CJ. The effects of fall-risk-increasing drugs on postural control: a literature review. Drugs \& aging. 2013;30(11):901-20.

2. Wöber C, Wöber Bingöl Ç, Karwautz A, Nimmerrichter A, Deecke L, Lesch OM. Postural control and lifetime alcohol consumption in alcohol-dependent patients. Acta Neurol Scand. 1999;99(1):48-53.

3. Rosenbloom MJ, Rohlfing T, O'Reilly AW, Sassoon SA, Pfefferbaum A, Sullivan EV. Improvement in memory and static balance with abstinence in alcoholic men and women: Selective relations with a change in brain structure. Psychiatry Res Neuroimaging. 2007;155(2):91-102.

4. Schmidt TP, Pennington DL, Cardoos SL, Durazzo TC, Meyerhoff DJ. Neurocognition and inhibitory control in polysubstance use disorders: Comparison with alcohol use disorders and changes with abstinence. J Clin Exp Neuropsychol. 2017; 39(1), 22-34.

5. Carlini EA, Garduróz JC, Noto AR, Nappo SA. I Household survey on the use of psychotropic drugs in Brazil: a study involving the 107 largest cities in the country-2001; São Paulo: Secretaria Nacional Antidrogas/Centro Brasileiro de Informações sobre Drogas Psicotrópicas, 2002.

6. Manthou E, Georgakouli K, Fatouros IG, Gianoulakis C, Theodorakis Y, Jamurtas AZ. Role of exercise in the treatment of alcohol use disorders. Biomed Rep. 2016;4(5):535-45.
7. Schmidt TP, Pennington DL, Durazzo TC, Meyerhoff DJ. Postural stability in cigarette smokers and during abstinence from alcohol. Alcohol Clin Exp Res 2014;38(6):1753-60.

8. Vancampfort D, Vandael H, Hallgren M, Probst M, Hagemann N, Bouckaert F, Van Damme T. Physical fitness and physical activity levels in people with alcohol use disorder versus matched healthy controls: a pilot study. Alcohol. 2019; 76, 73-79.

9. Pollock AS, Durward BR, Rowe PJ, Paul JP. What is balance? Clin Rehabil. 2000;14(4):402-6.

10. Fein G, Greenstein D. Gait and balance deficits in chronic alcoholics: No improvement from 10 weeks through 1-year abstinence. Alcohol Clin Exp Res. 2013;37(1):86-95.

11. Marczinski CA, Mearns CL. Automated assessment of alcohol-induced impairment of balance in male and female social drinkers. Exp Clin Psychopharmacol. 2020;28(2):131-6.

12. Fein G, Smith S, Greenstein D. Gait and balance in treatment naïve active alcoholics with and without a lifetime drug codependence. Alcohol Clin Exp Res. 2012;36(9):1550-62.

13. Schmidt PMS, Marques Giordani A, Garcia Rossi A, Luiz Cóser P. Evaluation of balance in alcoholics. Braz J Otorhinolaryngol. 2010; 76(2).

14. Moreira DA, Ganança MM, Caovilla HH. Static posturography in addicted to illicit drugs and alcohol. Braz J Otorhinolaryngol. 2012;78(5): 97-103.

15. Lima D, da Silva M, Júnior O, Lima L, Sampaio A, Luiz O. Association of leisure physical activity with consumption of alcoholic beverages in adults. Rev Bras Ativ Fis Saúde. 2017;22(6):576-83.

16. Hopkins W, Marshall S, Batterham A, Hanin J. Progressive statistics for studies in sports medicine and exercise science. Med Sci Sports Exerc. 2009;41(1):3.

17. World Health Organization. Global status report on alcohol and health-2014. World Health Organization; 2017.

18. Lipowski M, Szulc M, Buliński L. Physical activity among other health-related behaviors in treatment of alcoholism. J Sports Med Phys Fitness. 2015;55:231-40.

19. Malagodi B, Greguol M, Junior HS. Análise do equilíbrio corporal e aptidão física de indivíduos em tratamento para dependência química. Rev Bras Ativ Fis Saúde. 2018;23:1-9.

20. Abrantes AM, Blevins C, Lindsay C, Battle CL, Buman MP, Agu E, Stein M. Formative work in the development of a physical activity smartphone app targeted for patients with alcohol use disorders. Psychol Sport Exerc. 2019; 41, 162-171.

21. Vancampfort D, Vandael H, Hallgren M, Probst M, Hagemann N, Bouckaert F, Van Damme T. Physical fitness and physical activity levels in people with alcohol use disorder versus matched healthy controls: a pilot study. Alcohol. 2019;76:73-79.

22. Weinstock J, Farney MR, Elrod NM, Henderson CE, Weiss EP. Exercise as an adjunctive treatment for substance use disorders: Rationale and intervention description. J Subst Abuse Treat. 2017;72:40-7.

23. Bolbecker AR, Apthorp D, Martin AS, Tahayori B, Moravec L, Gomez KL, et al. Disturbances of postural sway components in cannabis users. Drug Alcohol Depend. 2018;190:54-61.

24. Sullivan EV, Rose J, Pfefferbaum A. Mechanisms of postural control in alcoholic men and women: biomechanical analysis of 
musculoskeletal coordination during quiet standing. Alcohol Clin Exp Res. 2010;34(3):528-37.

25. Silva D, Matias C, Bourne S, Nagel S, Machado A, Lobel D. Effects of chronic alcohol consumption on long-term outcomes of thalamic deep brain stimulation for essential tremor. J Clin Neurosci. 2016;31:142-6.

26. Vancampfort D, Hallgren M, Vandael H, Probst M, Stubbs B, Raymaekers S, Van Damme T. Test-retest reliability and clinical correlates of the Eurofit test battery in people with alcohol use disorders. Psychiatry research. 2019; 271, 208-213.

\section{Acknowledgement}

Coordenação de Aperfeiçoamento de Pessoal de Nível Superior (CAPES)

\section{Corresponding author}

Bruno Marson Malagodi

Universidade Estadual de Londrina, Educação Física, Rod. Celso Garcia Cid PR445 km 380, Londrina, PR.

Email: brunomarson32@gmail.com

Manuscript received on January 22, 2020

Manuscript accepted on April 13, 2020

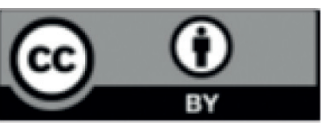

Motriz. The Journal of Physical Education. UNESP. Rio Claro, SP, Brazil - eISSN: 1980-6574 - under a license Creative Commons - Version 4.0 